\section{Ingenuity Pathway Analysis of miRNAs and mRNAs in Stored Platelets Identifies the Potential of miRNAs in Regu- lating Platelet Functions Rele- vant to Storage Lesions}

\section{Neetu Dahiya, Sandhya Kulkarni and Chintamani D Atreya*}

Laboratory of Cellular Hematology, Division of Hematology, Center for Biologics Evaluation and Research, US Food and Drug Administration, USA

\begin{abstract}
Under blood bank storage conditions platelets undergo physiological changes leading to storage lesions that affect platelet quality. Since small noncoding micrornas (miRNAs) are posttranslational regulators of cellular mRNA expression, we envisioned that miRNA targeting of mRNAs cause perturbations in stored platelets and affect their quality. In this report, differential miRNA and mRNA microarray data on day 0 , day 5 and day 9 stored human platelet samples were analyzed. The analysis identified 7 upregulated miRNAs (miR-320b, miR-1-3p, miR-214-3p, miR-197-3p, miR-129-5p, miR-183-5p and miR-292b-5p) that are known to regulate 35 mRNAs. The analysis further identified 68 differentially expressed miRNAs common to day 5 and day 9 of storage ( $p$-value $<0.05$ ). Ingenuity Pathway Analysis (IPA) based search for mRNAs that are potentially regulated by these miRNAs identified 17 mRNAs involved in platelet activation, 28 mRNAs associated with platelet aggregation, 12 mRNAs affecting platelet binding and 9 mRNAs involved in adhesion as well as 27 mRNAs implicated in cellular apoptosis. The IPA also assisted in identifying top 5 signaling pathways and network functions of platelets in storage. The results clearly identify the potential of miRNAs in regulating functions relevant to the platelet storage lesions, which provides a basis for further experimental verification.
\end{abstract}

Keywords: Ingenuity pathway analysis; microarray; miRNA; Networks; Platelets; Signaling pathway

*Corresponding author: Chintamani D Atreya, Laboratory of Cellular Hematology, Center for Biologics Evaluation and Research, Food and Drug Administration, 10903 New Hampshire Ave, Silver Spring, MD 20993, USA, Tel: +1 240-4028009; E-mail: chintamani.atreya@fda.hhs.gov

Citation: Dahiya N, Kulkarni S, Atreya CD (2016) Ingenuity Pathway Analysis of miRNAs and mRNAs in Stored Platelets Identifies the Potential of miRNAs in Regulating Platelet Functions Relevant to Storage Lesions. J Hematol Blood Transfus Disord 3: 012.

Received: September 27, 2016; Accepted: December 3, 2016; Published: December 19, 2016

\section{Introduction}

Platelets (PLTs) are one of the most important life-saving transfusion products. During storage under standard blood bank conditions, platelets start to lose their viability with time and as a consequence, platelets do undergo morphological and physiological changes during storage collectively known as Platelet Storage Lesion (PSL), which negatively impact their performance following transfusion.

MicroRNAs (miRNAs) are a type of small non-coding RNAs involved in posttranscriptional regulation of the genes (mRNAs) that they target. Role of miRNAs in platelets biosynthesis and presence of an active post-transcriptional miRNA mediated mRNA regulation has already been reported [1]. In addition, a number of platelet originated miRNAs have also been identified as possible disease biomarkers $[2,3]$. Platelets also release microparticles packed with miRNA-Ago2 RISC machinery capable of regulating gene expression and phenotype of the recipient cells [4,5]. More recently, Rowley et al., [6] reported the effect of miRNA processing on platelet functions using a Dicer1 deficient murine model. All these studies suggest that miRNAs play a role in platelet biology. However, none of these studies address whether perturbations in miRNAs and Messenger RNAs (mRNAs) do occur in platelets stored under blood bank conditions for transfusion, and the consequences of these perturbations on the quality of stored platelets.

Due to PSL development, both structure and some of the functions of platelets are compromised during storage. Our goal is to identify potential miRNA:mRNA interactions that are consequential to these changes. We have generated both miRNA and mRNA differential profiles and part of the data was reported $[7,8]$ and further analysis of this data constitutes present report. Here in this analysis, we performed a) IPA "MicroRNA target filter" tool to extract a list of potential miRNA-targeted mRNAs of stored platelets and, b) IPA "Core analysis" (In-silico analysis) to identify the potential functions of these $\mathrm{mR}$ NAs in stored platelets. Overall, this bioinformatics-based approach demonstrates that platelet miRNAs are capable of regulating mRNA functions relevant to PSL and provides confidence to the field to test each of these potential miRNA:mRNA interactions in the context of platelet storage quality towards improving the quality of platelets in storage.

\section{Methods}

\section{Platelet samples and storage}

Platelets samples collected from healthy donors at National Institutes of Health (NIH) blood bank were stored at $22^{\circ} \mathrm{C}$ in platelet shaker. The IRB approval for this study was exempted under FDA-RIHSC approved protocol \#03-120B. Samples were collected from the bag on day 0 , day 5 and day 9 of storage and leukocyte-reduced by using CD45 conjugated beads (Dynabeads, Life Technologies, Carlsbad, CA, USA) and samples were stored at $-80^{\circ} \mathrm{C}$.

\section{RNA extraction, quantification and quality assessment}

Total RNA was extracted using TRIZOL as per manufacturer's instruction (Life Technologies). Amount of RNA in each sample was 
quantified using NanoVue GE (GE, Pittsburgh, PA, USA) and quality of RNA samples were determined by gel-on-chip analysis using Agilent bioanalyzer.

\section{Microarrays and RT-qPCR}

MicroRNA profiling was performed using Affymetrix Gene chip miRNA 3.0 arrays. Samples collected from 4 different donors at three different time points were used for microRNA microarrays. For each array, $300 \mathrm{ng}$ of total RNA was used for performing the experiment. The mRNA profiling was done using HumanRef-8 Expression BeadChip (Illumina, Inc., San Diego, CA, USA) microarrays. Total RNAs extracted from two independent donors at three different storage time points were used for the profiling. RT-qPCR was done using TaqMan MicroRNA assays (Applied Biosystems, Foster City, CA, USA).

\section{MiRNA target filter analysis}

The detailed protocol for total RNA extraction from platelets stored for up to 9 days, miRNA and mRNA arrays and RT-qPCR are all as described previously [8]. Using microarrays, the differentially expressed miRNAs (fold cut off $>1.5$, p-value $<0.05$ ) and mRNA (fold cut off $>1.5$, p-value $<0.05$ ) during storage were identified. "MicroRNA target filter" function was applied in our ingenuity pathway analysis tool to determine potential targets of miRNAs differentially expressed on day 5 and day 9 compared to day 0 . The list of potential miRNA targets (i.e., mRNAs) compiled by IPA includes target genes predicted by different algorithms such as miRecords, target scan and experimental targets listed in tarbase (a database of experimental targets) and identified by Ingenuity Knowledge Base. The "MicroRNA target filter" tools provide a unique opportunity to find miRNA targets relevant to the biological context of interest (by performing miRNA and mRNAs profiling of the same samples) which can be very helpful in overcoming the complexity of miRNA target genes.

\section{Network functions and pathway analyses}

We populated a list of miRNAs differentially expressed on both day 5 and day 9 and extracted their potential target mRNAs using IPA tool. To find the mRNAs relevant to platelets in storage, mRNAs showing significant differential expression in platelets during storage were selected. To illustrate their functional role in platelets, these target genes were analyzed using IPA "Core analysis" function. The regulatory network functions identified are presented as a graph. The genes and their functional relation are represented as nodes and lines, respectively. The color of the nodes represents their up (red color) or downregulation (green color) status and their intensity represents the degree/extent of up or downregulation. The different shapes are indicative of the nature of the molecule based on their type of activity (i.e., cytokines, enzymes etc.). The top signaling pathways and network functions of the potential mRNA target genes using IPA were also analyzed. The significance of the pathways and network functions were determined based on the number of target genes identified and p-value $<0.05$.

\section{Results}

In our previous report [8] we found differential expression of 302 miRNAs in platelet samples during storage at $22^{\circ} \mathrm{C}$ (p-value $\left.<0.05\right)$. In this report, the principal component analysis of these significant miRNAs showed clear separation of samples into three distinct groups based on the days in storage (Figure 1). Of the 302 miRNAs, $68 \mathrm{miR}$ NAs have shown similar expression pattern on day 5 and day 9 , however the changes are more pronounced on day 9 compared to day 5
(Table 1). In order to understand the role of these differentially expressed miRNAs during storage, the mRNAs targeted for regulation by these miRNAs were determined. The mRNA profiling of two sets of the same RNA samples that were subjected to microRNA microarray profiling showed differential expression of 54 and 864 mRNAs on day 5 and day 9, respectively (Supplementary Table 1). To determine which mRNAs serve as potential targets of the differentially expressed common miRNAs, the Ingenuity Pathway Analysis tool was used. We searched for miRNA:mRNA pairs showing opposite expression pattern on different days of storage. The analysis resulted in identification of 1788 miRNA:mRNA pairs involving 59 miRNAs and 605 mRNAs showing negative correlation (Supplementary Table 2).

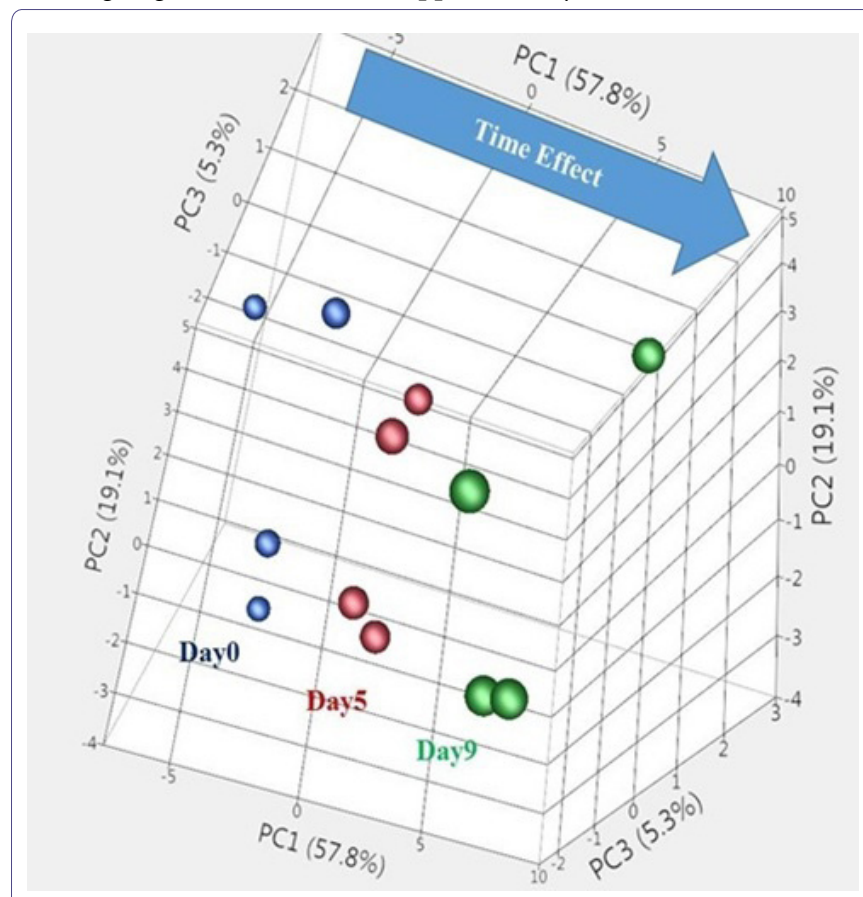

Figure 1: Principal Component Analysis (PCA) of platelet miRNAs differentially expressed $(p<0.05)$ on day 5 and day 9 of storage, compared to day 0 .

\begin{tabular}{|c|c|c|c|c|}
\hline \multirow{2}{*}{ MiRNA Name } & p-value & p-value & Fold-Change & $\begin{array}{c}\text { Fold-Change } \\
\text { (Day 9 vs. } \\
\text { Day 0) }\end{array}$ \\
\cline { 2 - 5 } (Day 5 vs. & $\begin{array}{c}\text { (Day 9 vs. } \\
\text { Day 0) }\end{array}$ & $\begin{array}{c}\text { Day 0) } \\
\text { Day }\end{array}$ & 3 & 5.1 \\
\hline hsa-miR-1224-5p & 0.025 & 0.004 & 4.6 & 7.7 \\
\hline hsa-miR-1225-5p & 0.014 & 0.003 & 1.7 & 1.8 \\
\hline hsa-miR-1226 & 0.017 & 0.011 & 9 & 24.4 \\
\hline hsa-miR-1231 & 0.015 & 0.003 & 3.4 & 4.2 \\
\hline hsa-miR-1254 & 0.01 & 0.005 & 4.7 & 14.1 \\
\hline hsa-miR-125b-1* & 0.011 & 0.001 & 1.5 & 2.1 \\
\hline hsa-miR-1270 & 0.012 & 0 & 2.8 & 9 \\
\hline hsa-miR-1275 & 0.013 & 0 & 1.6 & 1.5 \\
\hline hsa-miR-1287 & 0.023 & 0.045 & 2.5 & 3.5 \\
\hline hsa-miR-1292 & 0.026 & 0.008 & 1.6 & 3.6 \\
\hline hsa-miR-1304 & 0.046 & 0 & 3.5 & 6.5 \\
\hline hsa-miR-1469 & 0.042 & 0.009 & 3.6 & 8.2 \\
\hline hsa-miR-149* & 0.034 & 0.004 & 3.1 & 13 \\
\hline hsa-miR-150* & 0.039 & 0.001 & 1.5 & 1.8 \\
\hline hsa-miR-1538 & 0.034 & 0.009 & & \\
\hline
\end{tabular}




\begin{tabular}{|c|c|c|c|c|}
\hline hsa-miR-188-3p & 0.008 & 0.006 & -1.5 & -1.5 \\
\hline hsa-miR-1909* & 0.019 & 0.001 & 2.2 & 4.2 \\
\hline hsa-miR-193a-5p & 0.051 & 0.011 & 3.3 & 6 \\
\hline hsa-miR-197 & 0.015 & 0.002 & 1.5 & 1.9 \\
\hline hsa-miR-202* & 0.022 & 0.001 & -1.7 & -2.8 \\
\hline hsa-miR-2276 & 0.045 & 0.012 & 2 & 2.8 \\
\hline hsa-miR-23a* & 0.009 & 0.001 & 1.6 & 2.2 \\
\hline hsa-miR-3064-5p & 0.019 & 0.001 & 1.9 & 3.1 \\
\hline hsa-miR-31 & 0.028 & 0.034 & -1.9 & -1.8 \\
\hline hsa-miR-3162-5p & 0.006 & 0.001 & 2.6 & 3.7 \\
\hline hsa-miR-3163 & 0.033 & 0.003 & -1.6 & -2.2 \\
\hline hsa-miR-3177-3p & 0.028 & 0.01 & 2 & 2.5 \\
\hline hsa-miR-3185 & 0.034 & 0.007 & 4.6 & 9.5 \\
\hline hsa-miR-3187-3p & 0.046 & 0.004 & 3.3 & 8.6 \\
\hline hsa-miR-3195 & 0.043 & 0.002 & 2.8 & 9 \\
\hline hsa-miR-3605-5p & 0.023 & 0 & 1.6 & 3 \\
\hline hsa-miR-3646 & 0.005 & 0.002 & 1.5 & 1.7 \\
\hline hsa-miR-3663-3p & 0.024 & 0.003 & 6.4 & 18.1 \\
\hline hsa-miR-3679-5p & 0.029 & 0.003 & 2.6 & 5.1 \\
\hline hsa-miR-377* & 0.026 & 0.02 & 1.6 & 1.7 \\
\hline hsa-miR-3927 & 0.041 & 0.001 & -1.6 & -2.8 \\
\hline hsa-miR-3940-5p & 0.048 & 0.008 & 3 & 5.7 \\
\hline hsa-miR-432* & 0.018 & 0.015 & 1.6 & 1.6 \\
\hline hsa-miR-4462 & 0.053 & 0.011 & 2.5 & 3.9 \\
\hline hsa-miR-4463 & 0.039 & 0.005 & 3 & 5.8 \\
\hline hsa-miR-4486 & 0.026 & 0.003 & 5 & 14.8 \\
\hline hsa-miR-4516 & 0.034 & 0.014 & 3.6 & 4.9 \\
\hline hsa-miR-4523 & 0.001 & 0 & 1.6 & 2.2 \\
\hline hsa-miR-4530 & 0.029 & 0.009 & 3.3 & 5 \\
\hline hsa-miR-4532 & 0.007 & 0.001 & 6.8 & 24.9 \\
\hline hsa-miR-4651 & 0.03 & 0.01 & 3.7 & 5.7 \\
\hline hsa-miR-4674 & 0.026 & 0.003 & 4.2 & 10.2 \\
\hline hsa-miR-4689 & 0.026 & 0.014 & 5 & 6.5 \\
\hline hsa-miR-4695-5p & 0.02 & 0.003 & 3.4 & 6.3 \\
\hline hsa-miR-4707-5p & 0.025 & 0.006 & 4.1 & 7 \\
\hline hsa-miR-4734 & 0.03 & 0.009 & 4.2 & 6.9 \\
\hline hsa-miR-4741 & 0.016 & 0.005 & 5.4 & 8.8 \\
\hline hsa-miR-4745-5p & 0.023 & 0.006 & 4 & 6.8 \\
\hline hsa-miR-4791 & 0.01 & 0.003 & -2.6 & -3.4 \\
\hline hsa-miR-509-3-5p & 0.045 & 0.001 & -1.5 & -2.3 \\
\hline hsa-miR-510 & 0.022 & 0.006 & -1.8 & -2.2 \\
\hline hsa-miR-542-3p & 0.039 & 0.022 & -2.1 & -2.4 \\
\hline hsa-miR-548a-3p & 0.001 & 0 & -1.5 & -2.6 \\
\hline hsa-miR-548aa & 0.016 & 0 & -1.6 & -2.6 \\
\hline hsa-miR-548ac & 0.042 & 0.001 & -1.6 & -3.1 \\
\hline hsa-miR-548ae & 0.052 & 0.009 & -1.8 & -2.5 \\
\hline hsa-miR-548al & 0.043 & 0.042 & -2.1 & -2.1 \\
\hline hsa-miR-602 & 0.054 & 0.003 & 3.3 & 10.3 \\
\hline hsa-miR-603 & 0.036 & 0.004 & -1.8 & -2.7 \\
\hline hsa-miR-635 & 0.033 & 0.014 & -2.1 & -2.4 \\
\hline
\end{tabular}

\begin{tabular}{|c|c|c|c|c|}
\hline hsa-miR-642b & 0.004 & 0.001 & 4.4 & 6 \\
\hline hsa-miR-650 & 0.049 & 0.044 & 1.5 & 1.5 \\
\hline hsa-miR-943 & 0.03 & 0.001 & 1.6 & 2.5 \\
\hline
\end{tabular}

Table 1: List of 68 differentially expressed miRNAs common to day 5 and day 9 storage ( $p$-value $<0.05)$.

In the next step, we analyzed these 605 potential mRNAs using IPA "core analysis" to dissect their functional role in platelets. Interestingly, the mRNAs were found to be associated with several different signaling pathways, of which two pathways, 'actin cytoskeleton signaling' and 'integrin signaling' were already known to have important role in platelet biology. Ephrin receptor signaling, VEGF signaling and molecular mechanisms of cancer pathway were also among the top most affected pathways in platelets during storage. Table 2 lists top 5 signaling pathways involving these 605 mRNAs. With regards to the functional significance, in "molecular and cellular functions" category, hundreds of genes were associated with cell morphology, cellular assembly and organization, cellular movement, cellular growth and proliferation and cell death and survival (Table 3). Again, these represent all major morphological, physiological and functional changes that occur in platelets during storage.

\begin{tabular}{|c|c|c|}
\hline Pathway & Adj p-value & Number of Genes \\
\hline Actin Cytoskeleton Signaling & $<0.05$ & 29 \\
\hline Integrin Signaling & $<0.05$ & 28 \\
\hline Molecular Mechanisms of Cancer & $<0.05$ & 36 \\
\hline Ephrin Receptor Signaling & $<0.05$ & 23 \\
\hline VEGF Signaling & $<0.05$ & 17 \\
\hline
\end{tabular}

Table 2: IPA analysis of platelet miRNA-targeted mRNAs revealed top 5 cellular signaling pathways.

\begin{tabular}{|c|c|c|}
\hline Top Network Functions & Adj p-value & Number of Genes \\
\hline Cell Morphology & $<0.05$ & 239 \\
\hline Cellular Movement & $<0.05$ & 184 \\
\hline Cellular Assembly and Organization & $<0.05$ & 187 \\
\hline Cellular Growth and Proliferation & $<0.05$ & 282 \\
\hline Cellular Function and Maintenance & $<0.05$ & 244 \\
\hline
\end{tabular}

Table 3: IPA analysis of platelet miRNA-targeted mRNAs revealed top 5 network functions.

A number of genes altered during storage are known to have important functions in major physiological functions of platelets such as activation, adhesion, aggregation and binding (Figure 2). In addition to deregulation of genes important for maintaining the structure and function of platelets, we identified 27 genes (AKT1, BCL2L1, BCR, CASP2, CASP3, CBL, CD44, CDKN1A, CEBPB, DIABLO, EPOR, ETV6, FADD, FOXO3, HCLS1, IL7, IRS1, MAP2K1, NUMB, PRKCQ, PTPN6, RAC1, RAF1, SHC1, SYK, TAL1 and YY1) associated with apoptosis suggesting that platelets might be undergoing apoptosis during storage; a phenomenon until recently confirmed to occur only in nucleated cells (Figure 3 ).

In this study, we found down regulation of 35 mRNAs which are known targets (based on experimental targets in the IPA data analysis tool) for 7 miRNAs (miR-1-3p, miR-129-5p, miR-214-3p, miR-292b$5 \mathrm{p}$ and miR-320b), which are increased in platelets during storage (Figure 4). 


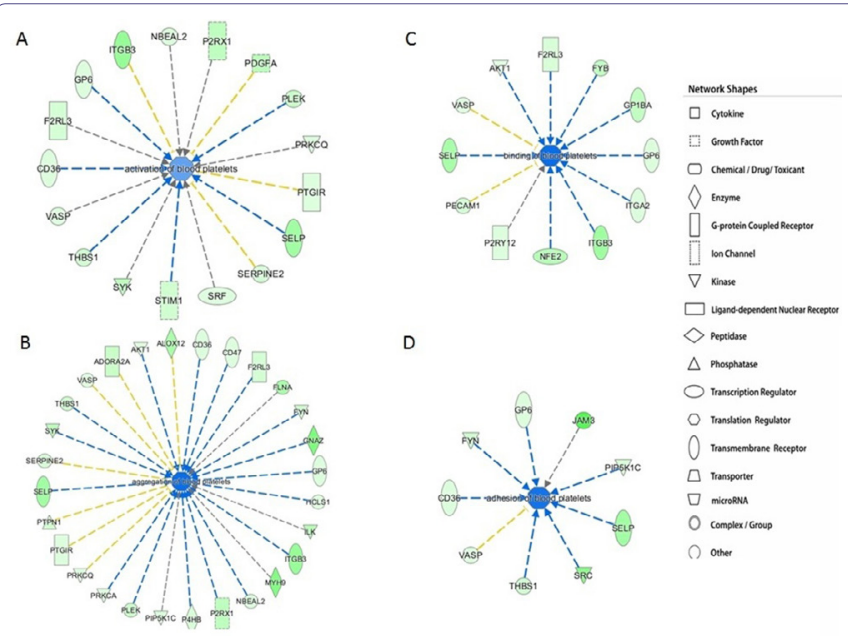

Figure 2: mRNAs potentially involved in regulating different platelet functions identified by IPA using the targets of 68 miRNAs. (A) Seventeen mRNAs implicated in platelet activation, (B) Twenty-eight mRNAs associated with aggregation, (C) Twelve mRNAs affecting platelet binding and (D) Nine mRNAs involved in adhesion.

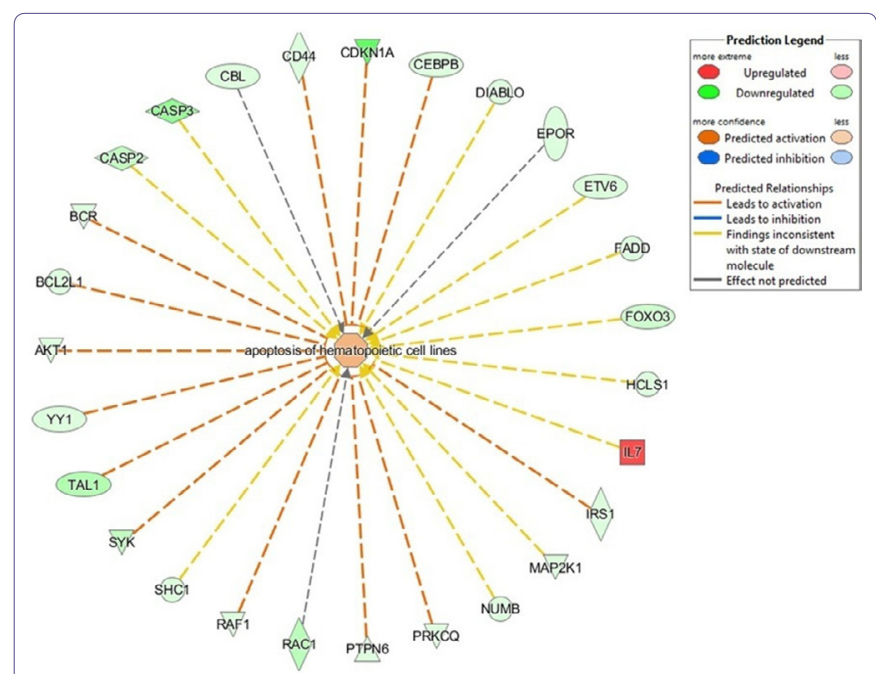

Figure 3: mRNAs potentially involved in apoptosis of hematopoietic cell lines identified by IPA using the targets of 68 miRNAs. The analysis identified 27 mRNAs implicated in apoptosis of hematopoietic cell lines that has relevance to platelet biology.

\section{Discussion}

Since mRNAs are subjected to regulation by miRNAs, it is important to assess the changes both in mRNA and miRNA expression levels of platelets during storage. The functional analysis of potential miRNA targets showing inverse correlation with mRNA expression identified several signaling pathways. The actin mediated cytoskeleton signaling and integrin signaling were the top most signaling pathways implicated in platelet functions such as aggregation, granule secretion and activation. Flaumenhaft et al., [9] also reported regulatory effect of actin cytoskeleton signaling on platelet granule secretion. In a previous study, 12 members of integrin signaling pathway were identified as contributors for storage lesion development [10]. Other significant pathways implicated in platelets during storage were focal adhesion pathways, IGF-1 signaling pathway and ephrin receptor signaling pathway. Integrin and focal adhesion pathways have been reported to be involved in early storage lesion in apheresis platelets [11]. IGF1 signaling pathway has been reported to regulate platelet activation

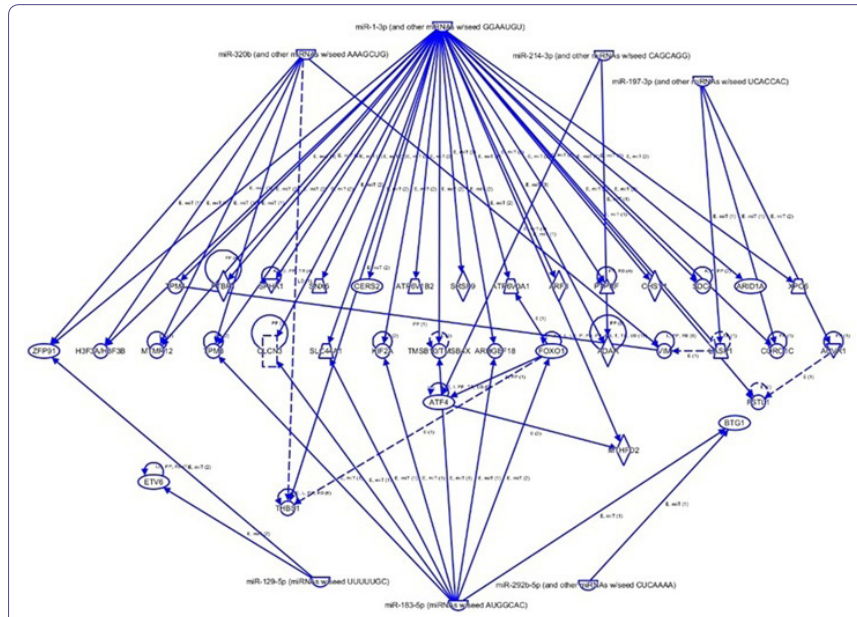

Figure 4: IPA network analysis of miRNAs and their known target mRNAs differentially expressed in platelets during storage. Seven miRNAs which were increased during storage and known to target 35 mRNAs are presented in this figure.

[12-13] and the ephrin receptor signaling pathway has been reported to regulate platelet granule secretion, Rap1 activation, platelet adhesion and aggregation [14-16].

The morphology of platelets do change during storage and platelet movement following transfusion into patients is critical as platelets are supposed to migrate rapidly to the site of damage to initiate formation of "platelet plug" to seal the damaged blood vessel and subsequent wound healing $[17,18]$. The change in expression levels of different genes identified in our study is able to reflect the changes in platelets morphology and physiological functions during storage. Since these molecular changes in terms of their ability to activate, aggregate and adhere as well as interact with each other are representative of the quality of platelets in storage, the differences in the expression levels of these genes may help develop platelet storage quality biomarkers predictive of their performance in platelet-transfused patients.

Apoptosis is an important process implicated in platelet storage lesion [19-20]. Our IPA analysis found a list of 27 apoptosis related genes. The list included genes such as CASP3 and BCL2L1 which are important players of apoptosis [21-23]. These candidate genes and the miRNAs that target these genes could be important in assessing the platelet quality during storage. The treatment of platelet concentrate with caspase inhibitor has been shown to increase the survival of the platelets [21]. Similar results were reported with use of antibodies against caspases [24]. The existence of apoptotic machinery in platelets and its alteration during storage provides another alternative for intervention to improve the quality of platelets during storage. The miRNAs targeting the genes of these apoptotic molecules could also serve as potential quality biomarkers of stored platelets.

In conclusion, the results reported here clearly identify the potential of miRNAs in regulating functions relevant to the platelet storage lesions. our analyses of the data we generated demonstrates that in fact there are several platelet signaling and functional pathways that are potentially subject to miRNA:mRNA interactions and 7 miRNAs that we identified to be upregulated (miR-320b, miR-1-3p, miR-214-3p, miR-197-3p, miR-129-5p, miR-183-5p and miR-292b-5p) could serve as potential platelets storage biomarkers. There is very limited information on functional role miRNAs in platelets. Of the seven miRNAs identified in our study only miR-320b has been studied in relation to 
Citation: Dahiya N, Kulkarni S, Atreya CD (2016) Ingenuity Pathway Analysis of miRNAs and mRNAs in Stored Platelets Identifies the Potential of miRNAs in Regulating Platelet Functions Relevant to Storage Lesions. J Hematol Blood Transfus Disord 3: 012.

its specific function in platelets. Activated platelets have been reported to release miR-320b in circulation where it has been found to regulate expression of Intercellular Adhesion Molecule-1 (ICAM-1) in endothelial cell [25]. Further experimental studies are required to assess the role of these miRNA:mRNA pairs.

\section{Acknowledgement}

ND was a recipient of a postdoctoral fellowship at the Center for Biologics Evaluation and Research (CBER) administered by the Oak Ridge Institute for Science and Education (ORISE) through an intra-agency agreement between the U.S. Department of Energy and the U.S. Food and Drug Administration.

\section{Authorship Contributions}

ND designed the study, performed experiments, analyzed data and wrote manuscript. CDA provided training to ND and also participated in designing the study and writing the manuscript. SK assisted ND in obtaining samples from NIH Blood Bank and ordering laboratory supplies and reagents needed for all experiments.

\section{Disclosure of Conflicts of Interest}

The authors declare that they have no conflicts of interest.

\section{References}

1. Edelstein LC, McKenzie SE, Shaw C, Holinstat MA, Kunapuli SP et al., (2013) MicroRNAs in platelet production and activation. J Thromb Haemost 11: 340350 .

2. Sondermeijer BM, Bakker A, Halliani A, de Ronde MW, Marquart AA, et al., (2011) Platelets in patients with premature coronary artery disease exhibit upregulation of miRNA340* and miRNA624*. PLoS One 6: 25946.

3. Fichtlscherer S, De Rosa S, Fox H, Schwietz T, Fischer A et al., (2010) Circulating microRNAs in patients with coronary artery disease. Circ Res 107 677-684.

4. Laffont B, Corduan A, Plé H, Duchez AC, Cloutier N et al., (2013) Activated platelets can deliver mRNA regulatory Ago2•microRNA complexes to endothelial cells via microparticles. Blood 122: 253-261.

5. Laffont B, Corduan A, Rousseau M, Duchez AC, Lee CH et al., (2016) Platelet microparticles reprogram macrophage gene expression and function. Thromb Haemost 115: 311-323.

6. Rowley JW, Chappaz S, Corduan A, Chong MM, Campbell R et al., (2016) Dicer1-mediated miRNA processing shapes the mRNA profile and function of murine platelets. Blood 127: 1743-1751.

7. Dahiya N, Sarachana T, Vu L, Becker KG, Wood WH 3rd et al., (2015) Platelet MicroRNAs: An Overview. Transfus Med Rev 29: 215-219.

8. Dahiya N, Sarachana T, Kulkarni S, Wood III WH, Zhang et al., (2016) miR570 interacts with mitochondrial ATPase subunit $g$ (ATP5L) encoding mRNA in stored platelets. Platelets 25: 1-8.

9. Flaumenhaft R, Dilks JR, Rozenvayn N, Monahan-Earley RA, Feng D et al., (2005) The actin cytoskeleton differentially regulates platelet alpha-granule and dense-granule secretion. Blood 105: 3879-3887.
10. Schubert P, Thon JN, Walsh GM, Chen CH, Moore ED et al., (2009) A signaling pathway contributing to platelet storage lesion development: targeting PI3-kinase-dependent Rap1 activation slows storage-induced platelet deterioration. Transfusion 49: 1944-1955.

11. Thiele T, luga C, Janetzky S, Schwertz H, Gesell Salazar M et al., (2012) Early storage lesions in apheresis platelets are induced by the activation of the integrin allb $\beta_{3}$ and focal adhesion signaling pathways. J Proteomics 76 : 297-315

12. Hers I (2007) Insulin-like growth factor-1 potentiates platelet activation via the IRS/PI3Kalpha pathway. Blood 110: 4243-4252.

13. Kim S, Garcia A, Jackson SP, Kunapuli SP (2007) Insulin-like growth factor-1 regulates platelet activation through PI3-Kalpha isoform. Blood 110: 42064213.

14. Tournoij E, Koekman CA, Du VX, Roest M, Ruijtenbeek R, et al., (2012) The platelet P2Y12 receptor contributes to granule secretion through Ephrin A4 receptor. Platelets 23: 617-625.

15. Prevost N, Woulfe D, Tanaka T, Brass LF (2002) Interactions between Eph kinases and ephrins provide a mechanism to support platelet aggregation once cell-to-cell contact has occurred. Proc Natl Acad Sci U S A 99: 9219-9224.

16. Prévost N, Woulfe DS, Tognolini M, Tanaka T, Jian W et al., (2004) Signaling by ephrinB1 and Eph kinases in platelets promotes Rap1 activation, platelet adhesion, and aggregation via effector pathways that do not require phosphorylation of ephrinB1. Blood 103: 1348-1355.

17. Nurden AT, Nurden P, Sanchez M, Andia I, Anitua E et al., (2008) Platelets and wound healing. Front Biosci 13: 3532-3548.

18. Packham MA (1994) Role of platelets in thrombosis and hemostasis. Can J Physiol Pharmacol 72: 278-284.

19. Leytin V, Freedman J (2003) Platelet apoptosis in stored platelet concentrates and other models. Transfus Apher Sci 28: 285-295.

20. Carvalho H, Alguero C, Santos M, de Sousa G, Trindade H et al., (2006) The combined effect of platelet storage media and intercept pathogen reduction technology on platelet activation/activability and cellular apoptosis/necrosis: Lisbon-RBS experience. Transfus Apher Sci 34: 187-192.

21. Shiri R, Yari F, Ahmadinejad M, Vaeli S, Tabatabaei MR et al., (2014) The caspase-3 inhibitor (peptide Z-DEVD-FMK) affects the survival and function of platelets in platelet concentrate during storage. Blood Res 49: 49-53.

22. Mutlu A, Gyulkhandanyan AV, Freedman J, Leytin V (2012) Activation of caspases- $9,-3$ and -8 in human platelets triggered by $\mathrm{BH} 3$-only mimetic $\mathrm{ABT}$ 737 and calcium ionophore A23187: caspase- 8 is activated via bypass of the death receptors. $\mathrm{Br} \mathrm{J}$ Haematol 159: 565-571.

23. Kile BT (2014) The role of apoptosis in megakaryocytes and platelets. $\mathrm{Br}$ Haematol 165: 217-226.

24. Piguet PF, Vesin C (2002) Modulation of platelet caspases and life-span by anti-platelet antibodies in mice. Eur J Haematol 68: 253-261.

25. Gidlöf O, van der Brug M, Ohman J, Gilje P, Olde B, et al., (2013) Platelets activated during myocardial infarction release functional miRNA, which can be taken up by endothelial cells and regulate ICAM1 expression. Blood 121: 3908-3917. 